\title{
The pattern electroretinogram in glaucoma: an evaluation by relative amplitude from the Bjerrum
}

\section{area}

\author{
ITSURO WATANABE, HIROYUKI IIJIMA, AND SHIGEO TSUKAHARA \\ From the Department of Ophthalmology, Yamanashi Medical College, Tamaho, Yamanashi, 409-38, Japan
}

\begin{abstract}
SUMMARY A new concept for evaluating the pattern electroretinogram (PERG) in glaucoma and its clinical application are described. The PERG was elicited by two different stimulus fields, namely, the whole central retinal area with a radius of $18^{\circ}$ and the paracentral ring area between the radius of $10^{\circ}$ and $18^{\circ}$. The amplitude of the PERG for each stimulus field and the ratio of them were analysed for 30 patients with glaucoma or ocular hypertension. Among these only a small number of eyes were detected as abnormal when we evaluated the amplitude itself either with the central stimulus or the paracentral ring stimulus, whereas the ratio of the two values was below normal in some cases of advanced glaucoma. We conclude that the ratio of the paracentral to central PERG is useful for detecting glaucoma.
\end{abstract}

The pattern electroretinogram (PERG) is an electrical retinal response elicited, not by the luminance change, but by the pattern stimuli in the visual field. Since the response has been considered to originate from the ganglion cell layer in the retina, ${ }^{\prime}$ many studies have been reported on the PERG's clinical application to glaucoma ${ }^{2-7}$ as well as optic nerve disorders. ${ }^{8-11}$ However, most of the reports on the PERG and glaucoma studied only the amplitude of the PERG elicited by the central stimulus field and generally found that patients with glaucoma have statistically smaller amplitudes than do control groups. ${ }^{56}$ In detecting the glaucomatous abnormality the amplitude of the PERG has not been proved to be sensitive, because it has large interindividual variation resulting in a wide overlap between glaucoma and normal control groups.

We previously reported that the PERG can be recorded from the paracentral ring area as well as from the central area. We also found that the ratio of the amplitudes from these two areas is less variable and more reliable than the amplitudes of themselves. ${ }^{12}$ Moreover, the ratio of the paracentral to central PERG is likely to be more sensitive in detecting glaucomatous changes, since the para-

Correspondence to Itsuro Watanabe, MD, Department of Ophthalmology, Yamanashi Medical College, Tamaho, Yamanashi, 409-38, Japan. central ring corresponds to the Bjerrum area, where early glaucomatous changes are believed to occur initially.

In this report we describe an effective method of analysing the PERG in glaucomatous eyes and its usefulness in detecting abnormality.

\section{Subjects and methods}

\section{SUBJECTS}

We studied 30 eyes of 30 patients with glaucoma or ocular hypertension $(\mathrm{OH})$ ranging in age from 16 to 80 years, mean 56 years. The detailed clinical findings are listed in Table 1.

In accordance with Kosaki's classification of glaucomatous field change they were divided into six groups. Stage I: Earliest stage of glaucoma without any field changes shown by kinetic Goldmann perimetry ( $a$ : normal field; $b$ :pathological field detected only when more precise method is used). Stage II: Early stage of glaucoma with abnormal field for isoptres I-4, I-3, I-2, and I-1, but without any abnormalities for an isoptre V-4 (a: normal for an isoptre $\mathrm{I}-4$, but abnormal for isoptres $\mathrm{I}-3, \mathrm{I}-2$, and $\mathrm{I}-1$; $b$ : abnormal for all I isoptres). Stage III: Middle (established or developed) stage of glaucoma, with abnormal field for an isoptre V-4, but with field loss not exceeding $1 / 2$ of the normal field for V-4 target 
Table 1 Clinical data

\begin{tabular}{|c|c|c|c|c|c|c|}
\hline No.lage/sex/eye & Diagnosis & $V A$ & $V F$ & $\begin{array}{l}\text { Full } \\
(\mu V)\end{array}$ & $\begin{array}{l}\text { Ring } \\
(\mu V)\end{array}$ & $\begin{array}{l}\text { Ring/full } \\
(\%)\end{array}$ \\
\hline 1/41/F/R & $\mathrm{OH}$ & $1 \cdot 2$ & I & $4 \cdot 53$ & $2 \cdot 32$ & $51 \cdot 2$ \\
\hline 2/45/M/R & $\mathrm{OH}$ & $1 \cdot 0$ & I & $2 \cdot 73$ & $1 \cdot 35$ & $49 \cdot 5$ \\
\hline $3 / 34 / F / R$ & $\mathrm{OH}$ & $1 \cdot 2$ & I & $4 \cdot 92$ & 1.95 & $39 \cdot 6$ \\
\hline 4/57/F/R & $\mathrm{OH}$ & $1 \cdot 0$ & I & $3 \cdot 13$ & $1 \cdot 25$ & $39 \cdot 9$ \\
\hline $5 / 64 / F / R$ & SEC & $1 \cdot 2$ & I & $4 \cdot 76$ & $1 \cdot 87$ & $39 \cdot 3$ \\
\hline 6/66/M/R & PACG & $1 \cdot 2$ & IIa & $2 \cdot 50$ & 0.93 & $37 \cdot 2$ \\
\hline 7/41/M/R & POAG & 0.7 & IIa & $2 \cdot 95$ & $1 \cdot 37$ & $46 \cdot 4$ \\
\hline $8 / 55 / F / R$ & POAG & $1 \cdot 2$ & IIa & $3 \cdot 59$ & $1 \cdot 64$ & $45 \cdot 7$ \\
\hline 9/52/F/L & POAG & $1 \cdot 2$ & IIb & $2 \cdot 31$ & 0.82 & $35 \cdot 5$ \\
\hline $10 / 80 / \mathrm{F} / \mathrm{L}$ & POAG & $1 \cdot 0$ & IIb & $2 \cdot 22$ & 0.82 & $36 \cdot 9$ \\
\hline $11 / 16 / \mathrm{M} / \mathrm{L}$ & POAG & $1 \cdot 2$ & IIb & $3 \cdot 00$ & $1 \cdot 15$ & $38 \cdot 3$ \\
\hline $12 / 70 / \mathrm{M} / \mathrm{L}$ & LTG & 0.9 & IIb & $1 \cdot 63$ & 0.56 & $34 \cdot 4$ \\
\hline 13/69/M/R & POAG & $1 \cdot 0$ & IIIa & $3 \cdot 35$ & $1 \cdot 32$ & $39 \cdot 4$ \\
\hline 14/45/M/R & POAG & $1 \cdot 2$ & IIIa & $4 \cdot 59$ & $2 \cdot 02$ & $44 \cdot 0$ \\
\hline 15/33/M/R & POAG & $1 \cdot 2$ & IIIa & $1 \cdot 87$ & 0.58 & $31 \cdot 0$ \\
\hline 16/43/M/R & POAG & $0 \cdot 8$ & IIIa & $2 \cdot 89$ & $1 \cdot 17$ & $40 \cdot 5$ \\
\hline $17 / 66 / \mathrm{M} / \mathrm{L}$ & POAG & $1 \cdot 2$ & IIIa & $1 \cdot 21$ & $0 \cdot 35$ & $28 \cdot 9$ \\
\hline 18/57/F/R & SEC & 0.9 & IIIa & $1 \cdot 71$ & 0.78 & $45 \cdot 6$ \\
\hline $19 / 72 / \mathrm{F} / \mathrm{L}$ & LTG & 0.8 & IIIa & $1 \cdot 32$ & 0.66 & $50 \cdot 0$ \\
\hline 20/61/F/R & LTG & $1 \cdot 2$ & IIIa & $3 \cdot 84$ & $1 \cdot 62$ & $42 \cdot 2$ \\
\hline 21/76/F/R & LTG & 0.7 & IIIa & $1 \cdot 79$ & 0.67 & $37 \cdot 4$ \\
\hline $22 / 39 / \mathrm{M} / \mathrm{L}$ & POAG & 0.9 & IIIb & $2 \cdot 00$ & $0 \cdot 68$ & $34 \cdot 0$ \\
\hline 23/26/M/R & POAG & 0.9 & IIIb & 2.50 & 0.68 & $27 \cdot 2$ \\
\hline $24 / 69 / \mathrm{M} / \mathrm{L}$ & POAG & 0.9 & IIIb & $1 \cdot 07$ & $0 \cdot 33$ & $30 \cdot 8$ \\
\hline 25/70/F/R & POAG & $0 \cdot 6$ & IIIb & $2 \cdot 03$ & 0.63 & $31 \cdot 0$ \\
\hline 26/71/M/L & POAG & $0 \cdot 8$ & IIIb & $2 \cdot 50$ & 0.85 & $34 \cdot 0$ \\
\hline $27 / 52 / \mathrm{M} / \mathrm{L}$ & SEC & $1 \cdot 0$ & IIIb & $2 \cdot 81$ & 0.86 & $30 \cdot 6$ \\
\hline $28 / 67 / \mathrm{F} / \mathrm{L}$ & LTG & $1 \cdot 0$ & IIIb & $3 \cdot 28$ & $1 \cdot 17$ & $35 \cdot 7$ \\
\hline 29/77/M/L & POAG & $0 \cdot 3$ & IV & $1 \cdot 40$ & $0 \cdot 51$ & $36 \cdot 4$ \\
\hline $30 / 69 / \mathrm{F} / \mathrm{L}$ & POAG & 0.7 & IV & $1 \cdot 52$ & $0 \cdot 54$ & $35 \cdot 5$ \\
\hline
\end{tabular}

$\mathrm{OH}=$ ocular hypertension. $\mathrm{SEC}=$ secondary glaucoma. $\mathrm{PACG}=$ primary angle closure glaucoma. $\mathrm{POAG}=$ primary open angle glaucoma. $\mathrm{LTG}=$ low tension glaucoma. $\mathrm{VA}=$ visual acuity $\mathrm{VF}=$ visual field, which is classified according to Kosaki's classification of glaucomatous field change.

For definitions of stages of glaucoma see text.

(V-4 field) ( $a$ : field loss (contraction) not exceeding $1 / 4$ of $\mathrm{V}-4$ field; $b$ : field loss more than $1 / 4$ but less than $1 / 2$ of V-4 field). Stage IV: Late stage of glaucoma, with field loss exceeding $1 / 2$ of $V-4$ field but with preserved macular field ( $a$ : field loss exceeding $1 / 2$ of V-4 field with preserved macular field; $b$ : macular field only). Stage V: Very late stage of glaucoma without macular field but with preserved field outside the macular field. Stage VI: End stage of glaucoma with loss of V-4 field. ${ }^{13}$

The intraocular pressure was controlled with pilocarpine, beta-blocking agents, epinephrine, carbonic anhydrase inhibitor, or combination of them.

\section{STIMULATION}

A television monitor placed $40 \mathrm{~cm}$ from the subject generated the checkerboard pattern stimuli, which were reversed rectangularly with a frequency of 10 cps. Each check size subtended $48 \times 39^{\prime}$ of visual angle. The mean luminance was $80 \mathrm{~cd} / \mathrm{m}^{2}$ and the

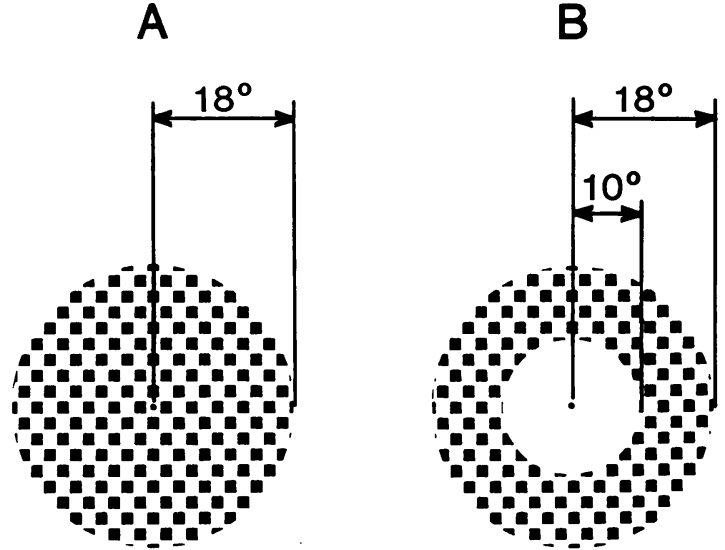

Fig. 1 A schema of the two stimulus fields. A: full pattern. B: ring pattern.

contrast was $94 \%$. Two different types of stimulus area were made available by placing one of the two masking boards in front of the TV monitor. The first allows presentation of the full pattern, that is, the checkerboard pattern inside the $18^{\circ}$ circle. The second with a central masking circle of $10^{\circ}$ of radius provided the ring pattern (Fig. 1).

\section{RECORDINGS}

The PERG was recorded with a corneal ERG-jet disposable electrode (Life-Tech Inc). The reference electrode was placed on the skin temporal to the test eye. The ground electrode was placed on the skin of the chin. The signals were amplified with a bandpass filter setting at $0 \cdot 5-30 \mathrm{~Hz}$ using a signal averager (MEB-5100, Nihon-Koden, Japan). We averaged 256 responses and removed artefacts from the data by an artefact rejection system.

MEASUREMENT AND ANALYSIS

Fig. 2 shows waveforms from a normal eye and from a glaucomatous eye. The amplitudes were measured from the second trough to the first peak as shown with arrows in Fig. 2. Three types of data, that is, the amplitude from $18^{\circ}$ circle pattern (full), the amplitude from $10^{\circ}$ to $18^{\circ}$ ring pattern (ring), and the ratio of ring to full were analysed. For each of them the normal mean with 2 standard deviations has been reported..$^{12}$

\section{Results}

Fig. 3a shows the distribution of the $18^{\circ}$ pattern stimulation for eyes with glaucoma and $\mathrm{OH}$, with the lower limit of the normal range. Almost all eyes in all groups had amplitudes larger than the lower limit of normal range. Fig. $3 b$ shows the distribution of the 
A

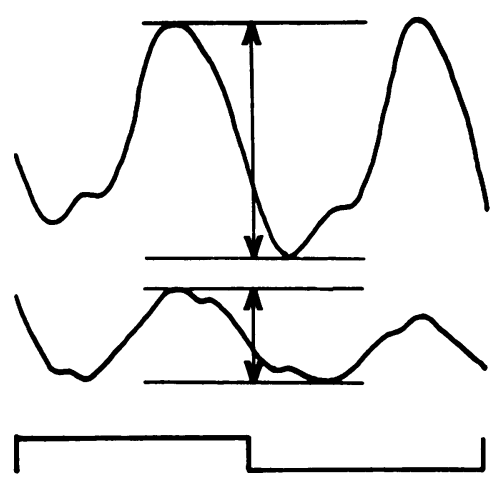

B

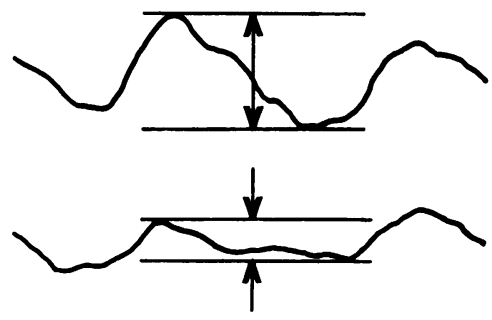

$50 \mathrm{~ms}$

Fig. 2 Waveforms from (A) a normal eye and (B) from a glaucomatous eye. The response for the full pattern is in the upper column and that from the ring pattern is in the middle column. The point of pattern change is indicated in the bottom trace.

$10^{\circ}$ to $18^{\circ}$ ring for the same subjects. Only a small number of eyes with more advanced glaucoma were plotted under the lower limit. Fig. 3c shows the distribution of the ratio of the ring to full stimulation. Eyes with fewer field defects were within the normal range, whereas a number of eyes of other groups were plotted under the lower limit. Exceptionally, most of the eyes in the classification of IIIa had a rather larger ratio. The records of their visual fields are shown in Figs. 4a-f.

\section{Discussion}

A histopathological investigation has shown that glaucomatous eyes even in the subclinical stage have suffered considerable loss of ganglion cells, ${ }^{14}$ which are the origin of the PERG. But, when we evaluate

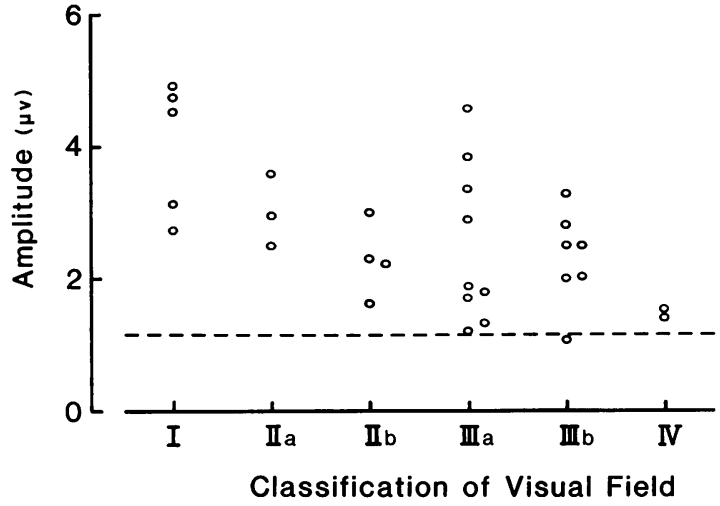

Fig. 3a

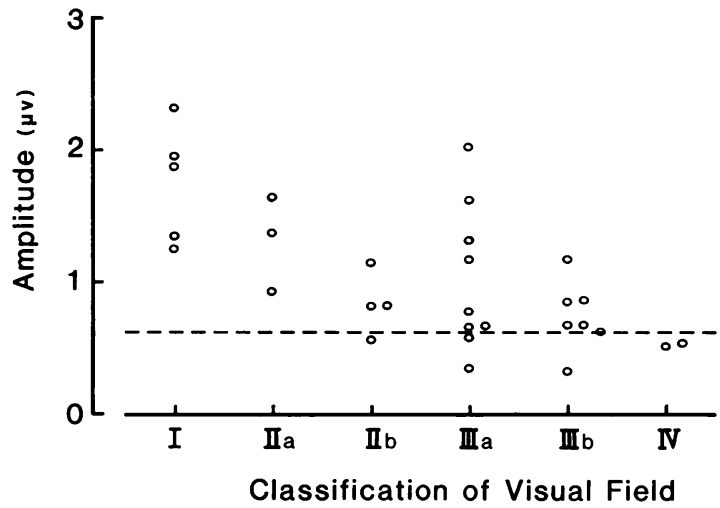

Fig. 3b

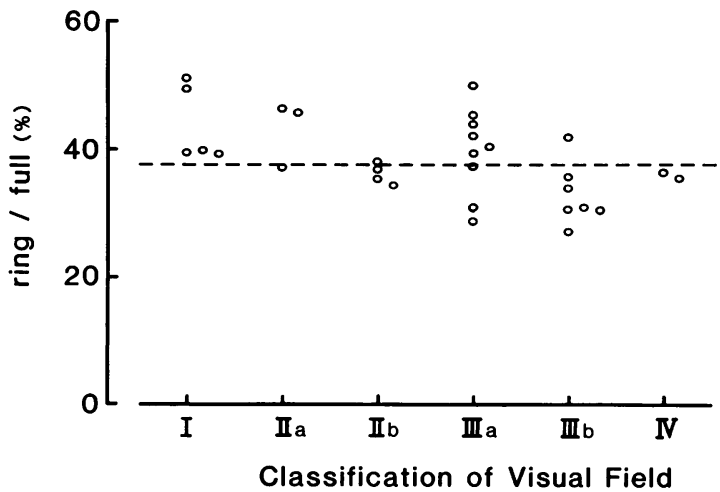

Fig. 3c

Fig. 3 The distribution of (a) the amplitudes of $18^{\circ}$ full stimulation, (b) the amplitudes of $10^{\circ}$ to $18^{\circ}$ ring stimulation, and (c) the ratio of ring to full among various stages of glaucomatous field change. The broken line indicates the lower limit of the normal range. 
Matg sintel is

NO. 13

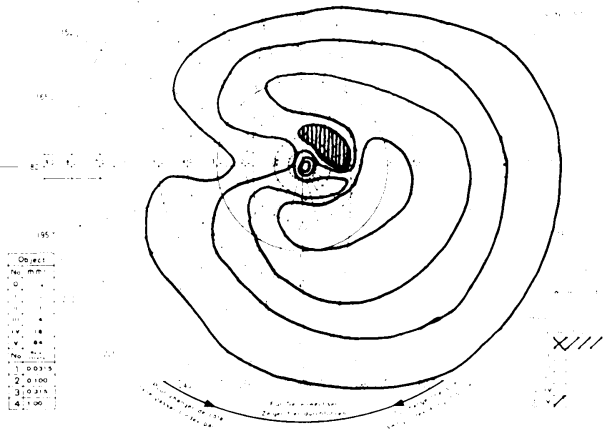

Fig. 4 a

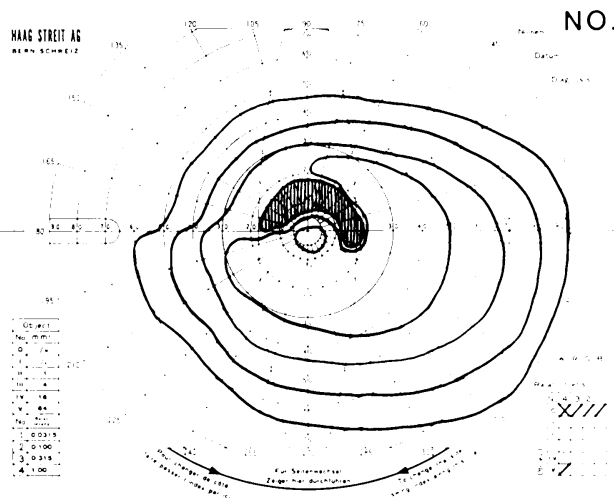

Fig. $4 b$

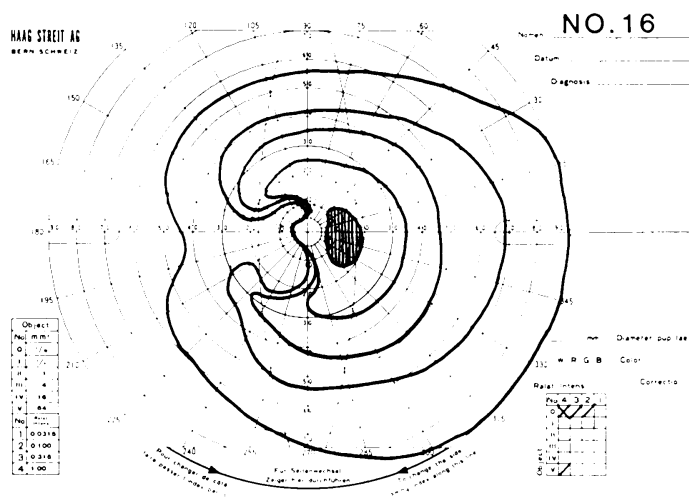

Fig. 4c

Fig. 4 The visual fields of (a) case 13, (b) case 14, (c) case 16, (d) case 18, (e) case 19, and (f) case 20. Their ratios of ring to full were exceptionally large (Fig. 3c), which could be ascribed to the pattern of their field being hemianoptic rather than having Bjerrum defects.

the PERG amplitude of the $18^{\circ}$ circle, no significant abnormality could be detected in most of the glaucomatous eyes. Since the glaucomatous field

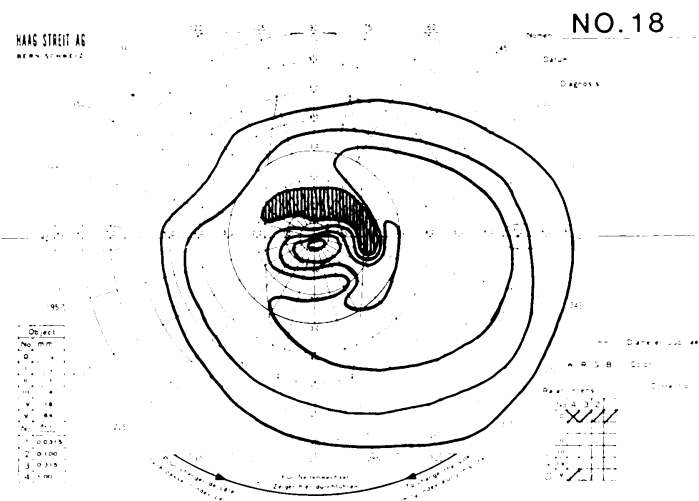

Fig. $4 d$

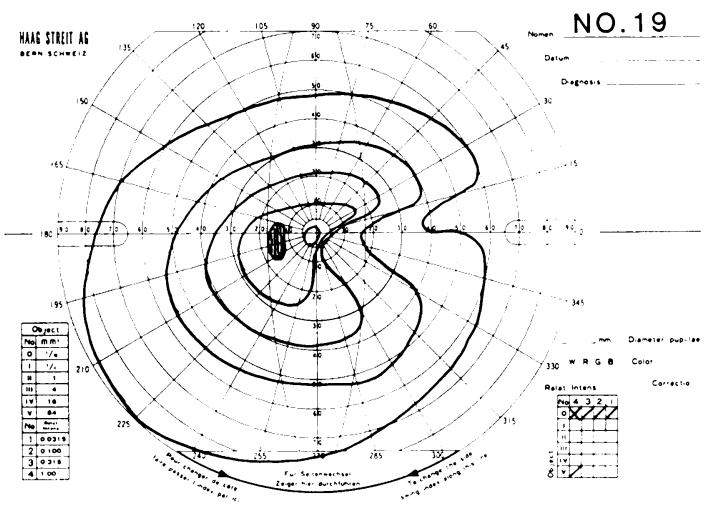

Fig. $4 \mathrm{e}$

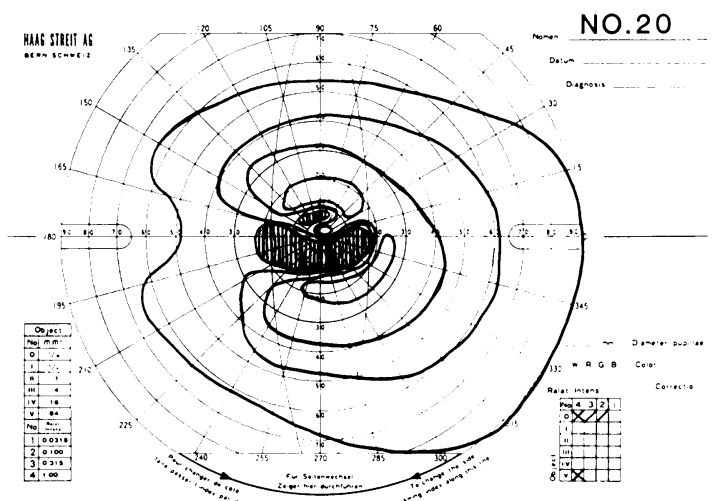

Fig. $4 f$

defect arises in the Bjerrum region, it would be expected that in many patients a selective stimulus in this area would be more effective than that in the whole central field. From this working hypothesis we devised a ring shaped stimulus field corresponding to the Bjerrum area and found that the sensitivity of detecting the glaucomatous abnormality was slightly 
improved, though it was still unsatisfactory (Fig. 3b). Such insensitivity is thought to derive from the large variation in the amplitude of the PERG recorded in normal as well as in glaucomatous eyes. The variation is the result of various factors, such as pupil size, which is affected by the use of pilocarpine drops, unstable fixation, eye movement, different conductance round the eyeball, and others. But these factors have the same effects on both the amplitudes of the PERG elicited by the $18^{\circ}$ full stimulation and $10^{\circ}$ to $18^{\circ}$ ring stimulation. It is expected that the effects would be cancelled in the evaluation of the ratio of the two values. In fact, the ratio of ring to full was shown to have a narrower normal range, with the result that most of the glaucomatous eyes with documented field change are plotted under the normal lower limit. This concept of evaluation by the ratio of two values has also been adopted in other fields of ocular electrophysiology. Examples are the $\mathrm{L} / \mathrm{D}$ ratio ${ }^{15}$ and the relative base value $\mathrm{e}^{16}$ in the electrooculogram, and $\mathrm{a} / \mathrm{b}$ ratio $^{17}$ in the electroretinogram, whose usefulness has already been established.

Some exceptional cases do exist, especially in classification IIIa (Fig. 3c). Their fields are hemianoptic rather than having Bjerrum defects (Fig. 4), and with hemianoptic field defects both responses from full and ring stimulus fields are equally reduced, resulting in a more or less normal ratio of ring to full. Additional methods to cover the cases with these rather atypical glaucomatous field defect patterns will be devised later.

On the whole, however, the ratio of ring to full has proved useful for detecting glaucomatous change in comparison with the amplitude of the PERG alone. We recommend that the PERG evaluation of patients with potential glaucoma should use the ratio of Bjerrum retinal area to the overall posterior retina to achieve the most effective diagnostic results.

The authors thank Dr J R Heckenlively for help with the preparation of the paper.

\section{References}

1 Maffei L, Fiorentini A. Electroretinographic responses to alternating gratings before and after section of the optic nerve. Science 1981; 211: 953-55.

2 Bobak P, Bodis-Wollner I, Harnois C, et al. Pattern electroretinograms and visual-evoked potentials in glaucoma and multiple sclerosis. Am J Ophthalmol 1983; 96: 72-83.

3 Wanger P, Persson HE. Pattern-reversal electroretinograms in unilateral glaucoma. Invest Ophthalmol Vis Sci 1983; 24: 749-53.

4 Papst N, Bopp M, Schnaudigel OE. Pattern electroretinogram and visually evoked cortical potentials in glaucoma. Graefes Arch Clin Exp Ophthalmol 1984; 222: 29-33.

5 Ringens PJ, Vijfvinkel-Bruinenga S, van Lith GHM. The pattern-elicited electroretinogram. I. A tool in the early detection of glaucoma? Ophthalmologica 1986; 192: 171-5.

6 Trick GL. PRRP abnormalities in glaucoma and ocular hypertension. Invest Ophthalmol Vis Sci 1986; 27: 1730-6.

7 May JG. Ralston JV, Reed JI, Van Dyk HJL. Loss in patternelicited electroretinograms in optic nerve dysfunction. $A m J$ Ophthalmol 1982; 93: 418-22.

8 Dawson WW, Maida TM, Rubin ML. Human pattern-evoked retinal responses are altered by optic atrophy. Invest Ophthalmol Vis Sci 1982; 22: 796-803.

9 Seiple W, Price MJ, Kupersmith M, Siegel IM, Carr RE. The pattern electroretinogram in optic nerve disease. Ophthalmology 1983; 90: 1127-32.

10 Persson HE, Wanger P. Pattern-reversal electroretinograms and visual evoked cortical potentials in multiple sclerosis. $\mathrm{Br} J$ Ophthalmol 1984; 68: 760-4.

11 Ringens PJ, van Lith GHM, Poel Hvd. The pattern-elicited electroretinogram. II. Retinal responses in retrobulbar neuritis. Ophthalmologica 1986; 192: 217-9.

12 Watanabe I, Iijima $\mathrm{H}$, Satoh S. A new stimulus method of pattern-elicited electroretinography to detect early changes in glaucoma. Folia Ophthalmol Jpn 1986; 37: 1098-102.

13 Kosaki H, Inoue Y. A new classification of stages of chronic glaucomas. Nippon Ganka Gakkai Zasshi 1972; 76: 1258-67.

14 Quigley HA, Hohman RM, Addicks EM, Massof RW, Green WR. Morphologic changes in the lamina cribrosa correlated with neural loss in open-angle glaucoma. Am J Ophthalmol 1983; 95: 673-91.

15 Arden GB, Barrada A, Kelsey JH. New clinical test of retinal function based upon the standing potential of the eye. $B r J$ Ophthalmol 1962; 46: 449-67.

16 Iijima H, Watanabe I, Satoh S, Seto C. Effects of retinal detachment surgery on standing potential. Doc Ophthalmol 1986; 63: 157-63.

17 Henkes HE, Electro-ophthalmology for the clinician. Jpn J Ophthalmol 1981; 25: 267-79.

Accepted for publication 28 January 1988. 